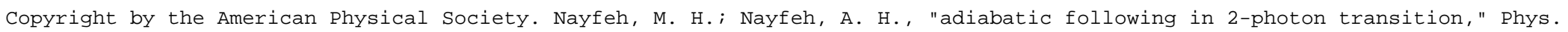
Rev.A 15, 1169 (1977); DOI: http://dx.doi.org/10.1103/PhysRevA.15.1169

PH Y I C A L R EVIEW A

VOLUME 15, NUMBER 3

M A R C H 1977

\title{
Adiabatic following in two-photon transition
}

\author{
Munir H. Nayfeh \\ Oak Ridge National Laboratory, ${ }^{*}$ Oak Ridge, Tennessee 37830
}

\author{
Ali H. Nayfeh
}

Virginia Polytechnic Institute and State University, Blacksburg, Virginia 24061

(Received 2 August 1976)

\begin{abstract}
The coherent interaction of two smoothly varying, near-resonant, two-photon pulses with a three-level system can be described by "two-photon damped Bloch equations" which are analogous to those for a one-photon transition in a two-level system except for the presence of a two-photon coupling and a frequency shift. These equations are solved for the cases $\gamma_{1}, \gamma_{2} \ll \Omega, \gamma_{1}=\gamma_{2}$, and $\gamma_{2} k^{2} \epsilon^{4} / \Omega^{2}, \gamma_{1} \ll \Omega$, where $\gamma_{1}$ and $\gamma_{2}$ are the atomic energy and phase relaxation widths, respectively, and $\Omega$ is the Rabi frequency. The leading contribution to the refractive index is intensity dependent, caused by the level shifts inherent in multiphoton processes; it includes a relaxation dependent part which is important at times shorter than $\gamma_{1}^{-1}$. The second-order contributions depend on the square of the intensity and the time-integrated square of the intensity. The latter contribution, which is relaxation dependent, causes line asymmetry at the long-wavelength wing; it consists of a term proportional to $\gamma_{2}-\gamma_{1}$ and only important at early times and a term proportional to $2 \gamma_{2}-\gamma_{1}$.
\end{abstract}

More interest has been generated recently in coherent multiphoton transitions in many-level systems..$^{1-5}$ Coherent two-photon propagations, exhibiting self-induced transparency when twice the propagating frequency is resonant with a twolevel system, have been analyzed, ${ }^{1,2}$ and the oneand two-photon resonant fields were shown to obey together an area theorem. ${ }^{2}$ Tan-no et al.$^{3}$ reported the observation of coherent propagations based on a two-photon transition which involved the interaction of two optical pulses of different frequencies with a gaseous three-level system. Further, self-transparency in a resonant two-photon absorption with a two-level system was observed. ${ }^{4}$

In this work, we consider the effect of relaxation on the response of a three-level system to a smoothly varying near-resonant two-photon field. This system, when the intermediate state is sufficiently far from resonance, can be described by the following "two-photon Bloch equations" which are analogous to those for a one-photon transition in a two-level system, except for the presence of a two-photon coupling and a frequency shift ${ }^{5}$ :

$$
\begin{aligned}
& \frac{d u}{d t}+\gamma_{2} u=-\Delta(t) v, \\
& \frac{d v}{d t}+\gamma_{2} v=\Delta(t) u+k \epsilon^{2}(t) w, \\
& \frac{d w}{d t}+\gamma_{1} w=-k \epsilon^{2}(t) v-\gamma_{1},
\end{aligned}
$$

where

$$
\begin{aligned}
& \Delta(t)=\Delta_{0}+s_{1} \epsilon_{1}^{2}+s_{2} \epsilon_{2}^{2}, \quad \Delta_{0}=\omega_{1}+\omega_{2}-\omega_{12}, \\
& s_{1}=\frac{\mu_{01}^{2}}{4 \hbar^{2} \Delta \omega_{0}}, \quad s_{2}=\frac{\mu_{12}^{2}}{4 \hbar^{2} \Delta \omega_{0}}, \quad k=\frac{\mu_{01} \mu_{12}}{2 \hbar^{2} \Delta \omega_{0}},
\end{aligned}
$$

$$
\epsilon^{2}=\epsilon_{1}(t) \epsilon_{2}(t), \quad \Delta \omega_{0}=\left|\omega_{1}-\omega_{01}\right|=\left|\omega_{2}-\omega_{12}\right|,
$$

$\Delta \omega_{0}$ is the off-resonant frequency; $\omega_{01}$ and $\omega_{12}$ are the eigenfrequencies of the levels; $\epsilon_{1}(t)$ and $\epsilon_{2}(t)$ are the amplitudes of the electric-field modes defined by $E=\epsilon_{1}(t) \cos \omega_{1} t+\epsilon_{2}(t) \cos \omega_{2} t ; u$ and $v$ are the dipole dispersive and absorptive components, respectively; $w$ is the inversion; $\mu_{01}$ and $\mu_{12}$ are the dipole moments of the levels in units of $\hbar$; and $\gamma_{1}=1 / T_{1}$ and $\gamma_{2}=1 / T_{2}$ are the atomic energy and phase relaxation widths, respectively.

Damping effects have been incorporated in the above equations phenomenologically by introducing a longitudinal relaxation time $T_{1}$ of the upper level and a homogeneous transverse relaxation time $T_{2}$. The relaxation effects of the intermediate level may be neglected if it is sufficiently far from resonance.

An adiabatic-following model based on the above approximation was used to derive and explain parametric generation processes. ${ }^{6}$ Power-dependent nonlinear susceptibilities were obtained, and pulse propagation was studied. However, in the above study, relaxation of the atomic levels was neglected. In this paper we determine the effect of relaxation on the model and calculate the relaxationdependent contributions to the nonlinear refractive index.

When the amplitudes of the fields vary slowly with time, one can use the method of multiple scales ${ }^{7}$ to determine an approximation to the general solutions of Eqs. (1)-(3). Alternatively, one can first reduce these equations to three uncoupled third-order equations with time-dependent coefficients. To accomplish this, we first eliminate the variable $v$ from Eq. (3) by multiplying Eq. (3) by $k \epsilon^{2}\left(d / d t+\gamma_{2}\right) / k \epsilon^{2}$ and then substituting for 
$\left(d / d t+\gamma_{2}\right) v$ from Eq. (2). The result is

$$
\begin{aligned}
& {\left[\left(\frac{d}{d t}+\gamma_{2}-\frac{d}{d t} \ln k \epsilon^{2}\right)\left(\frac{d}{d t}+\gamma_{1}\right)+k^{2} \epsilon^{4}\right] w} \\
& =-k \epsilon^{2} \Delta u-k \epsilon^{2}\left(\frac{d}{d t}+\gamma_{2}\right) \frac{\gamma_{1}}{k \epsilon^{2}} .
\end{aligned}
$$

The variable $u$ can be eliminated from Eq. (4) by multiplying it by $k \epsilon^{2} \Delta\left(d / d t+\gamma_{2}\right) / k \epsilon^{2} \Delta$ and substituting for $\left(d / d t+\gamma_{2}\right) u$ from Eq. (1) and for $v$ from Eq.
(3). We neglect terms of the order of $d^{2} \ln \epsilon / d t^{2}$, $(d \ln \epsilon / d t)\left(d \ln \epsilon^{2} \Delta / d t\right)$, and $(d \ln \epsilon / d t)^{2}$ in the homogeneous part of the equations and terms of the order of $d \ln \epsilon / d t$ in the sources. Terms of the order of $d \ln \epsilon / d t$ are kept in the homogeneous part because their integrals over time determine the solution; consequentially, these terms are not negligible for nonconstant-field amplitudes even as the rate of change of the amplitudes becomes arbitrarily small. Using a similar procedure for Eq. (1), one can show that the results for $w$ and $u$ are

$$
\begin{array}{r}
\begin{array}{r}
\frac{d^{3}}{d t^{3}}+\left(2 \gamma_{2}+\gamma_{1}-\frac{d}{d t} \ln \left(k^{2} \epsilon^{4} \Delta\right)\right) \frac{d^{2}}{d t^{2}}+\left(\left(\gamma_{1}+\gamma_{2}\right)^{2}-\gamma_{1}^{2}+\Omega^{2}-\left(\gamma_{2}+\gamma_{1}\right) \frac{d}{d t} \ln \left(k^{2} \epsilon^{4} \Delta\right)\right) \frac{d}{d t}+\gamma_{1}\left(\Delta^{2}+\gamma_{2}^{2}\right) \\
\left.-\left(k^{2} \epsilon^{4}+\gamma_{1} \gamma_{2}\right) \frac{d}{d t} \ln \left(k \Delta \epsilon^{2}\right)+\left(\gamma_{2}+\frac{d}{d t} \ln k^{2} \epsilon^{4}\right) k^{2} \epsilon^{4}\right] w=-\gamma_{1}\left(\Delta^{2}+\gamma_{2}^{2}\right)
\end{array} \\
{\left[\begin{array}{r}
\frac{d^{3}}{d t^{3}}+\left(2 \gamma_{2}+\gamma_{1}-\frac{d}{d t} \ln k \epsilon^{2} \Delta^{2}\right) \frac{d^{2}}{d t^{2}}+\left(\left(\gamma_{1}+\gamma_{2}\right)^{2}-\gamma_{1}^{2}+\Omega^{2}-\left(\gamma_{1}+\gamma_{2}\right) \frac{d}{d t} \ln \Delta-2 \gamma_{2} \frac{d}{d t} \ln k \epsilon^{2} \Delta\right) \frac{d}{d t}+\gamma_{1}\left(\gamma_{2}^{2}+\Delta^{2}\right) \\
\left.+\gamma_{2} k^{2} \epsilon^{4}+\left(\gamma_{2}^{2}+\Delta^{2}\right) \frac{d}{d t} \ln k \epsilon^{2} \Delta+\left(2 \Delta^{2}-\gamma_{1} \gamma_{2}\right) \frac{d}{d t} \ln \Delta\right] u=\gamma_{1} \Delta k \epsilon^{2},
\end{array}\right.}
\end{array}
$$

where

$$
\Omega^{2}(t)=\Delta^{2}(t)+k^{2} \epsilon^{4} .
$$

The general solution of Eq. (5) can be easily reduced to quadratures by assuming the logarithmic time derivative of the field $d(\ln \mu \epsilon) / d t$, which is a measure of the pulse width, to be small compared with the Rabi frequency $\Omega$. To this end, we write

$$
\left(\frac{d}{d t}+g_{3}\right)\left(\frac{d}{d t}+g_{2}\right)\left(\frac{d}{d t}+g_{1}\right) w=-\gamma_{1}\left(\Delta^{2}+\gamma_{2}^{2}\right)
$$

where

$$
\begin{aligned}
& g_{1}+g_{2}+g_{3}=2 \gamma_{2}+\gamma_{1}-\frac{d}{d t} \ln \left(k^{2} \epsilon^{4} \Delta\right) \\
& \frac{d}{d t}\left(2 g_{1}+g_{2}\right)+g_{1}\left(g_{2}+g_{3}\right)+g_{2} g_{3} \\
& =\Omega^{2}+\left(\gamma_{1}+\gamma_{2}\right)^{2}-\gamma_{1}^{2}-\left(\gamma_{2}+\gamma_{1}\right) \frac{d}{d t} \ln \left(k^{2} \epsilon^{4} \Delta\right), \\
& \frac{d}{d t}\left(g_{2} g_{1}\right)+\frac{d^{2} g_{1}}{d t^{2}}+g_{3} \frac{d g_{1}}{d t}+g_{1} g_{2} g_{3} \\
& =\gamma_{1}\left(\Delta^{2}+\gamma_{2}^{2}\right)-\left(k^{2} \epsilon^{4}+\gamma_{1} \gamma_{2}\right) \frac{d}{d t} \ln \left(k^{2} \epsilon^{3} \Delta\right) \\
& +k^{2} \epsilon^{4}\left(\gamma_{2}+\frac{d}{d t} \ln k^{2} \epsilon^{4}\right)
\end{aligned}
$$

Since $d(\ln \Delta \epsilon) / d t$ is small compared with $\Omega$, we can write

$$
g_{i}=G_{i}+\delta_{i} \text { with }\left|\delta_{i}\right| \ll\left|G_{i}\right| .
$$

With this approximation, - we have reduced the dif- ferential equations (8)-(11) to two sets of algebraic equations for the eigenfrequencies $G_{1}, G_{2}$, and $G_{3}$ and for the amplitude functions $\delta_{1}, \delta_{2}$, and $\delta_{3}$. The equation for $G_{i}$ is cubic. In the most general case, no simple factorization occurs and a cubic equation must be solved. However, in some cases the cubic equation can be factored. These include the case of equal decay widths, the case where the decay widths are smaller than or equal to $O(d \ln \epsilon / d t)$, and the case of small fields and small $\gamma_{1}$. The ef fect of relaxation on adiabatic following in twolevel systems was discussed recently..$^{9-11}$ Using the present method, the first author solved the one-photon Bloch equation under the above conditions. ${ }^{9}$

When $\gamma_{1}=\gamma_{2}=\gamma$ (which is a reasonable assumption for strong collisions),

$$
\begin{aligned}
& g_{1}=\gamma-d[\ln (\Delta / \Omega)] / d t, \\
& g_{2}=\gamma-i \Omega-d\left(\ln k \epsilon^{2}\right) / d t, \\
& g_{3}=\gamma+i \Omega-d\left[\ln \left(k \epsilon^{2} \Omega\right)\right] / d t .
\end{aligned}
$$

Substituting for the $g_{n}$ in Eq. (8) and solving for $w$, one finds that the general solution consists of two homogeneous solutions which oscillate with the Rabi frequency, a homogeneous, nonoscillatory solution, and a particular solution. For adiabatic fields and an atom that is initially in the ground state, the oscillatory solutions vanish and $w$ takes the simple form

$$
w=-\frac{\Delta}{\Omega} e^{-\gamma t}-\gamma \frac{\Delta}{\Omega} e^{-\gamma t}\left(\int_{-\infty}^{t} \frac{\Omega e^{\gamma \tau}\left(\gamma^{2}+\Delta^{2}\right)}{\Delta\left(\gamma^{2}+\Omega^{2}\right)} d \tau\right) .
$$


The population inversion does not have any powerdependent decay. As $\gamma \rightarrow 0$,

$$
w \rightarrow-\Delta / \Omega
$$

in agreement with the calculation of Ref. 6 .

It is interesting to compare the above results with the results for two-level systems. ${ }^{9}$ The first contribution in Eq. (16) is identical in form to the corresponding contribution in two-level systems, except that the detuning $\Delta$ is a function of time due to the frequency shift and the Rabi frequency $\Omega$ is modified by the two-photon coupling and the frequency shift. The presence of the shift causes a shift in the crossing point of the inversion $[\Delta(t)=0]$. However, the integral contribution form is different from that for two-level systems because $\Delta$ is a function of time in three-level systems. In the limit of constant $\Delta$, the two-photon integral contribution reduces to that of the one-photon case.

When $\gamma_{1}$ and $\gamma_{2}$ are small but different, one can easily show that the population inversion for the case of adiabatic following is given by

$$
\begin{aligned}
w=-\frac{\Delta(t)}{\Omega(t)} e^{-\gamma_{1} t-q(t)} & -\gamma_{1} e^{-\alpha(t)} \frac{\Delta(t)}{\Omega(t)} \\
& \times \int_{-\infty}^{t} \frac{\Delta(\tau)}{\Omega(\tau)} e^{\gamma_{1}(\tau-t)+q(\tau)} d \tau,
\end{aligned}
$$

where

$$
q(t)=\left(\gamma_{2}-\gamma_{1}\right) \int_{-\infty}^{t} \frac{k^{2} \epsilon^{4}}{\Omega^{2}} d t
$$

We note that the inversion has an induced damping $q(t)$. For weak fields, the lowest-order term in $q(t)$ depends on the integrated square of the intensity which is an indication of the two-photon process. For sufficiently strong fields, $q(t)$ has no field dependence, and the inversion is damped by the phase relaxation $\gamma_{2}$ only as in the one-photon transition.

In addition to the line shift, which is inherent in two-photon processes due to the involvement of virtual intermediate levels, there is a relaxationdependent line asymmetry due to the buildup of the pulsed field. The asymmetry is analogous to that in two-level systems ${ }^{9}$; in this case, it is modified as a result of the field dependence of the line shift in multiphoton processes. To see this, we derive an expression for the refractive index of three-level systems in the weak-field limit. Under the same conditions used to derive Eq. (18), one can easily show that the solution of Eq. (6) is

$$
u=\frac{k \epsilon^{2}}{\Omega} e^{-\gamma_{1} t-q(t)}\left(1+\gamma_{1} \int_{-\infty}^{t} \frac{\Delta(\tau)}{\Omega(\tau)} e^{\gamma_{1} \tau+q(\tau)} d \tau\right) .
$$

When $\gamma_{1}=\gamma_{2}=0$, the solution for $u$ reduces to $k \epsilon^{2} / \Omega$, in agreement with the result of Ref. 6 . Since the refractive index of the medium is related to $u$ by

$$
n=1+2 \pi N k \hbar u \epsilon^{-2},
$$

it is given for weak fields by

$$
\begin{aligned}
n-1=-\frac{2 \pi N k^{2} \hbar}{\Delta_{0}}[ & \left(1-\frac{s_{1} \epsilon_{1}^{2}+s_{2} \epsilon_{2}^{2}}{\Delta_{0}}+\frac{\left(s_{1} \epsilon_{1}^{2}+s_{2} \epsilon_{2}^{2}\right)^{2}}{\Delta_{0}^{2}}-\frac{1}{2} \frac{k^{2} \epsilon^{4}}{\Delta_{0}^{2}}\right)\left(1+e^{-\gamma_{1}}{ }^{t}\right) \\
& \left.-\frac{2 \gamma_{2}-\gamma_{1}}{2} \int_{-\infty}^{t} \frac{k^{2} \epsilon^{4}(\tau)}{\Delta_{0}^{2}} e^{\gamma_{1}(t-\tau)} d \tau-\left(\gamma_{2}-\gamma_{1}\right) e^{-\gamma_{1} t} \int_{-\infty}^{t} \frac{k^{2} \epsilon^{4}(\tau)}{\Delta_{0}^{2}} d \tau\right]+O\left(\epsilon^{6}\right) .
\end{aligned}
$$

We note that the lowest-order contribution to the induced refractive index is intensity dependent. It is equal to $2 \pi N k^{2} \hbar \Delta_{0}^{-2}\left(s_{1} \epsilon_{1}^{2}+s_{2} \epsilon_{2}^{2}\right)\left[1+\exp \left(-\gamma_{1} t\right)\right]$ which is inversely proportional to the square of the detuning of the transition. The nextorder contribution is $\pi N k^{2} \hbar \Delta_{0}^{-3}\left[k^{2} \epsilon^{4}-2\left(s_{1} \epsilon_{1}^{2}\right.\right.$ $\left.\left.+s_{2} \epsilon_{2}^{2}\right)^{2}\right]\left[1+\exp \left(-\gamma_{1} t\right)\right]$; part of this contribution is due to the two-photon level shift. The last two contributions depend on the integrated square of the intensity. These contributions cause asymmetry in the refractive index at the long-wavelength wing of the transition due to the accumulation of the square of the intensity at later times. We note that for pulses of the same total energy, the asymmetry depends on the pulse length. For long times the term proportional to $\gamma_{2}-\gamma_{1}$ vanishes, while the term proportional to $2 \gamma_{2}-\gamma_{1}$ increases with increasing times. Thus, it is expected that there exists a pulse length for which the asymmetry is maximum.

All of these contributions are dependent on the relaxation. In the absence of relaxation (i.e., $\gamma_{1}=\gamma_{2}=0$ ), it follows from Eq. (22) that

$$
\begin{aligned}
n-1= & -\frac{4 \pi N k^{2} \hbar}{\Delta_{0}} \\
& \times\left(1-\frac{s_{1} \epsilon_{1}^{2}+s_{2} \epsilon_{2}^{2}}{\Delta_{0}}+\frac{2\left(s_{1} \epsilon_{1}^{2}+s_{2} \epsilon_{2}^{2}\right)^{2}-k^{2} \epsilon^{4}}{2 \Delta_{0}^{2}}\right) .
\end{aligned}
$$

When the relaxation is due entirely to spontaneous emission (i.e., $2 \gamma_{2}=\gamma_{1}$ ), the second contribution in Eq. (22) vanishes. In this case, the two-photon level shifts contribute to the asymmetry of the line to $O\left(\epsilon^{4}\right)$ at early times only. At times larger than the relaxation time $T_{1}$, it follows from Eq. (22) that 


$$
\begin{aligned}
n-1= & -\frac{2 \pi N k^{2} \hbar}{\Delta_{0}} \\
& \times\left(1-\frac{s_{1} \epsilon_{1}^{2}+s_{2} \epsilon_{2}^{2}}{\Delta_{0}}+\frac{2\left(s_{1} \epsilon_{1}^{2}+s_{2} \epsilon_{2}^{2}\right)-k^{2} \epsilon^{4}}{2 \Delta_{0}^{2}}\right. \\
& \left.-\frac{\left(2 \gamma_{2}-\gamma_{1}\right)}{2} \int_{-\infty}^{t} \frac{k^{2} \epsilon^{4}(\tau)}{\Delta_{0}^{2}} e^{\gamma_{1}(\tau-t)+q(\tau)} d \tau\right) .
\end{aligned}
$$

The "two-photon Bloch equations" can also be solved using the above method when $\gamma_{1} \ll \Omega$ and $\gamma_{2} k^{2} \epsilon^{4} \ll \Omega^{3}$. In this case the condition on the relaxation $\gamma_{2}$ is less restrictive. For weak fields, $\gamma_{2}$ could be taken large enough so that the rate-equation limit is attained.

In conclusion, we have been able to derive an adiabatic-following solution for a three-level system interacting with two smooth pulses. Moreover, we derived an expression for the refractive index of the medium which is relaxation dependent. Two contributions to the refractive index depend on the time-integ rated square of the intensity and cause line asymmetry.
*Operated by Union Carbide Corp. for the U. S. Energy Research and Development Administration.

${ }^{1}$ E. M. Belenov and I. A. Polucktov, Zh. Eksp. Teor. Fiz. 56, 1407 (1969) [Sov. Phys.-JETP 29, 754 (1969)]. ${ }^{2}$ M. Takatsuji, Phys. Rev. A 4 , 808 (1971).

${ }^{3} \mathrm{~N}$. Tan-no, K. Yokoto, and H. Inaba, Phys. Rev. Lett. 29, 1211 (1972).

${ }^{4}$ T. L. Gvardzhaladze, A. Z. Grasyuk, and V. A. Kovalenko, Zh. Eskp. Teor. Fiz. 64, 446 (1973) [Sov. Phys.JETP 37, 227 (1973)].

${ }^{5}$ N. Tan-no, K. Yokoto, and H. Inaba, J. Phys. B $\underline{8}, 339$ (1975).
${ }^{6}$ D. Grischkowsky, M. M. T. Loy, and P. F. Liao, Phys. Rev. A 12, 2514 (1975).

${ }^{7}$ A. H. Nayfeh, Perturbation Methods (Wiley-Interscience, New York, 1973), Chap. 6.

${ }^{8}$ M. G. Payne and M. H. Nayfeh, Phys. Rev. A $\underline{13}, 595$ (1976).

${ }^{9}$ M. H. Nayfeh, Phys. Rev. A 14, 1304 (1976).

${ }^{10}$ R. H. Lehmberg and J. Reintjes, Phys. Rev. A 12 , 2574 (1975).

${ }^{11}$ M. H. Nayfeh and A. H. Nayfeh, J. Appl. Phys. $\underline{47}$, 2528 (1976). 